\title{
INHALT \\ von Band 142
}

KRYSTYNA BARTOL, The Importance of Appropriateness. Rethinking the Definition of Nomos . . . . . . . . . . . . . . . . . . . . . . . 300

INGEBORG BENECKE-DeLtAaglia und ERNST GÜNTHER SCHMIDT, Zum 150. Todestag von Gottfried Hermann. Stücke aus dem Nachlaß . . . . . . . . . 335

Bruno BleckMAnN, Regulus bei Naevius: Zu Frg. 50 und 51 Blänsdorf . . . . 61

William M. Calder III, Gottfried Hermann to Thomas Gaisford. An Unpublished Letter . . . . . . . . . . . . . . . . . . . . . . . . . . 359

SERGIO CASALI, Ovidio e la preconoscenza della critica. Qualche generalizzazione a partire da Heroides $14 \ldots \ldots \ldots \ldots \ldots \ldots . \ldots . \ldots 94$

Johannes CHRistes, Beatus ille. Eine Konjektur und ihr Ertrag . . . . . 279

JOHANNES CHRISTES, Lucilius senex - vetus historia - Epilog zu XXVI-XXX: drei alte Fragen neu verhandelt $\ldots \ldots \ldots \ldots \ldots \ldots \ldots \ldots \ldots \ldots \ldots$

Jaime B. Curbera, Onomastics and River-Gods in Sicily $\ldots \ldots \ldots \ldots \ldots . \ldots 2$

AleXANDer Kleinlogel, Beobachtungen zu den Thukydidesscholien II . . . . . 11

Theokrtos Kouremenos, Aristotle on Syllogistic and Mathematics . . . . . 220

Peter Kruschwitz, Überlegungen zum Text der Hedyphagetica des Ennius . . . 261

David Payne Kubiak, Epic and Comedy in Prudentius' Hymn to St. Eulalia.

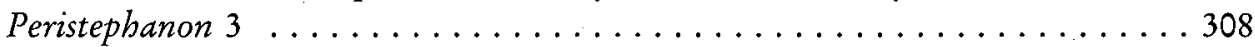

ANDREI LeBeDEv, The Justice of Chiron (Titanomachia, Fr. 6 and 11 B.) . . . 3

HarTMUTT LePPIN, Eduard Nordens Berufung nach Greifswald: Handlungsspielräume im „System Althoff“" ..................... 162

WALTER Ludwig, Das bessere Bildnis des Gelehrten . . . . . . . . . 123

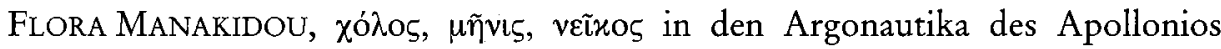
Rhodios. Reminiszenzen und Umkehrungen der homerischen Epen im helle-

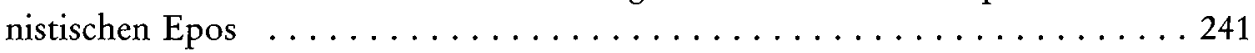

Erich POTZ, Appians Klio dichtet. Die Curio-Episode bei Appian (E 2, 44,

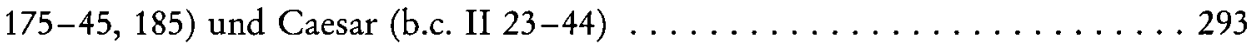

JoHn Richmond, The Relationship of Vindob. 277 and Paris. Lat. 8071 . . . . 80

GERSON SCHADE, Ennius und Archestratos . . . . . . . . . . . . . 275

JENS-UwE SCHMIDT, Ares und Aphrodite - der göttliche Ehebruch und die theo-

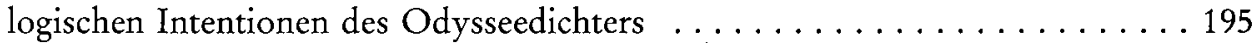

Robert SCHMiel, Repetition in Nonnos' Dionysiaca ............... 326

KurT SIer, Platon, Protagoras 345 a $1-b 8 \ldots \ldots \ldots \ldots \ldots \ldots \ldots \ldots \ldots$

Jürgen Tubach, Der Beginn der Sintflut nach Berossos $\ldots \ldots \ldots \ldots \ldots \ldots 114$ 


\section{Miszellen}

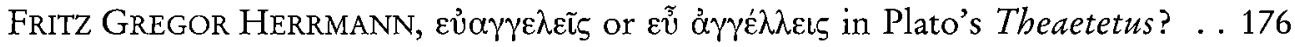
RaIner Jakobi, At Thetis. Argumentum in Statii Achilleidem . . . . . . . . . 369

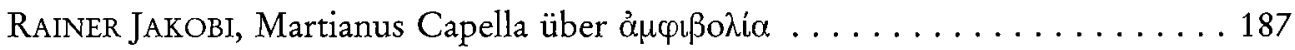

MARTIN KORENJAK, Der Rezitator als verprügelter Koch: zu einem locus vexatus bei Persius . . . . . . . . . . . . . . . . . . . . . . . . . . . . . 365

Klaus LenNartZ, Catull 29 und 29 a? . . . . . . . . . . . . . . . . . . 361

Klaus LenNaRTZ, Catull 58, 5 und die Wortgeschichte von magnanimus . . . . 363

WOLFGANG LUPPE, Vermischung zweier Euripides-Hypotheseis? . . . . . . . . 173

Werner J. SCHNEIdER, Homers Helden und die Tyrannenmörder von Athen. Zum Text von Philostrat, Ep. 56 (57) p. 251.26/27 Kayser $^{2}$. . . . . . . . . 181 WolfGANG CHRISTIAN SCHNEIDER, Ein neu entdecktes Fragment einer LiviusHandschrift in Darmstadt . . . . . . . . . . . . . . . . 185

Berichtigungen zu Philologus 142 (1998) $1 \ldots \ldots \ldots \ldots$. . . . . . . . 374 


\title{
PHILOLOGUS
}

Zeitschrift für antike Literatur und ihre Rezeption

HERAUSGEBER

Joachim Ebert (Halle), Widu-Wolfgang Ehlers (Berlin), Fritz Jürß (Berlin),

Ernst Günther Schmidt (Leipzig), Peter Lebrecht Schmidt (Konstanz), Bernd Seidensticker (Berlin)

\author{
REDAKTIONSBEIRAT \\ István Borzsák (Budapest), William M. Calder III (Urbana), \\ Benedetto Marzullo (Roma), Adelina Piatkowski (București), \\ P. H. Schrijvers (Leiden), Heikki Solin (Helsinki), \\ Hanna Szelest (Warszawa)
}

Geschäftsführende Herausgeber des Bandes 142: Widu-Wolfgang Ehlers, Bernd Seidensticker

Redaktion: Dietlind Schieferdecker

Band $142 \cdot 1998 \cdot$ Heft 2

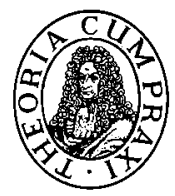

Akademie Verlag 
Die Zeitschrift PHILOLOGUS dient der Erforschung des griechisch-römischen Altertums in seinen geistig-kulturelien Formen und deren Wirkungsgeschichte. Die philologischen Untersuchungen betreffen sprachlich-begriffliche und textkritische Probleme ebenso wie allgemeine Fragen der Literatur, Religion, Philosophie und Wissenschaft der Antike.

\section{Bezugsmöglichkeiten}

Bitte richten Sie Ihre Bestellungen an

R. Oldenbourg Verlag, Zeitschriftenservice, Postfach 801360 , D-81613 München,

Telefon: (0 89) 450512 29/3 99, Telefax: (0 89) 45051333.

Zeitschrift „Philologus“

Herausgeber: Prof. em. Dr. J. Ebert, Prof. Dr. W. W. Ehlers, Prof. Dr. F. Jürß, Prof. Dr. E. G. Schmidt, Prof. Dr. P. L. Schmidt, Prof. Dr. B. Seidensticker.

Geschäftsführende Herausgeber des Bandes 142: Prof. Dr. W. W. Ehlers, Prof. Dr. B. Seidensticker.

Anschrift der Redaktion: Redaktion „Philologus", Berlin-Brandenburgische Akademie der Wissenschaften, Jägerstr. 22/23, D-10117 Berlin; Telefon: (0 30) 20370681.

Verlag: Akademie Verlag GmbH, Mühlenstr. 33-34, D-13187 Berlin; Telefon: (0 30) 4788 93 51, Telefax: (030)4788 9357; http://www.oldenbourg.de/akademie-verlag/; Bank: Bayerische Vereinsbank AG, München, Kto.-Nr.: 50841 812, BLZ 700202 70; Deutsche Bank AG, München, Kto.-Nr.: 2250 710, BLZ 70070010.

Geschäftsführer: Dr. Gerd Giesler, Johannes Oldenbourg.

Anzeigenannahme: Suzan Hahnemann, R. Oldenbourg Verlag, Telefon: (0 89) 4505 13 40; Telefax: (0 89) 45051266.

Satz: Druckhaus „Thomas Müntzer“ GmbH, D-99947 Bad Langensalza.

Druck und buchbinderische Weiterverarbeitung: GAM MEDIA GmbH, D-10969 Berlin.

Erscheinungsweise: Die Zeitschrift erscheint jährlich in einem Band mit 2 Heften. Jahresbezugspreis 1998 Inland DM 362,-; Ausland DM 375,-. Einzelheft DM 209,-. Studenten: Inland DM 102,-; Ausland DM 110,- jeweils zuzüglich Versandkosten.

Das Abonnement verlängert sich jeweils um ein weiteres Jahr, falls es nicht 8 Wochen vor Ablauf eines Kalenderjahres gekündigt wird.

Authorization to photocopy items for internal or personal use, or the internal or personal use of specific clients, is granted by Akademie Verlag GmbH, provided that the base fee of US $\$ 10.00$ per copy, plus US $\$ .25$ per page is paid directly to copyright Clearance Center, 27 Congress Street, SALEM, MA 01970) USA. For those organizations that have been granted a photocopy license by CCC, a separate system of payment has been arranged.

The fee code for users of the Transactional Reporting Service is: 0031-7985/98 \$10.00+.25.

Urheberrecht: Alle Rechte vorbehalten, insbesondere die der Übersetzung. Kein Teil dieser Zeitschrift darf in irgendeiner Form - durch Fotokopie, Mikrofilm oder irgendein anderes Verfahren - ohne schriftliche Genehmigung des Verlages reproduziert oder in eine von Maschinen, insbesondere von Datenverarbeitungsanlagen verwendbare Sprache übertragen oder übersetzt werden.

(C) 1998 by Akademie Verlag GmbH. Printed in the Federal Republic of Germany.

Der Akademie Verlag ist ein Unternehmen der R. Oldenbourg-Gruppe.

Gedruckt auf alterungsbeständigem Papier.

Dieses Heft entbält folgende Beiträge: Vandenhoeck \& Ruprecht: Hypomnemata 